\title{
The combined effects of real or simulated microgravity and red light photoactivation on plant root meristematic cells
}

\author{
Miguel A. Valbuena ${ }^{1,2 \#}$, Aránzazu Manzano ${ }^{1 \#}$, Joshua P. Vandenbrink ${ }^{3}$, Veronica Pereda- \\ Loth $^{4}$, Eugenie Carnero Diaz ${ }^{2}$, Richard E. Edelmann ${ }^{5}$, John Z. Kiss ${ }^{3}$, Raúl Herranz ${ }^{1 *}$ F. \\ Javier Medina ${ }^{1 *}$ \\ ${ }^{1}$ Centro de Investigaciones Biológicas (CSIC), Madrid, E28040, Spain \\ ${ }^{2}$ Institut Systématique, Evolution, Biodiversité (ISYEB), Museum National d'Histoire \\ Naturelle, CNRS, Sorbonne Université, EPHE.57 rue Cuvier CP39, 75005 Paris, France \\ ${ }^{3}$ Department of Biology, University of North Carolina at Greensboro, Greensboro, NC \\ 27402, USA \\ ${ }^{4}$ Université de Toulouse III UPS, GSBMS-AMIS, Faculty of Medicine Rangeuil, Toulouse, \\ France \\ ${ }^{5}$ Department of Biology, Miami University, Oxford, OH 45056, USA
}

${ }^{\#}$ These authors contribute equally to this work.

* Corresponding authors:

Dr.Raúl Herranz, r.herranz@csic.es

Dr.F. Javier Medina, fjmedina@cib.csic.es

Ramiro de Maeztu,9, E-28040, Madrid Spain

Phone: +349183731 12, Fax: +34915360432 


\section{Abstract}

Main conclusion Red light is able to compensate for deleterious effects of microgravity on root cell growth and proliferation. Partial gravity combined with red light produces differential signals during early plant development.

Light and gravity are environmental cues used by plants throughout evolution to guide their development. We have investigated the cross-talk between phototropism and gravitropism under altered gravity in space. The focus was on the effects on the meristematic balance between cell growth and proliferation, which is disrupted under microgravity in the dark. In our spaceflight experiments, seedlings of three Arabidopsis thaliana genotypes, namely the wild type and mutants of phytochrome A and B, were grown for six days, including red light photoactivation for the last two days. Apart from the microgravity and the $1 g$ on-board control conditions, fractional gravity (nominally $0.1 g, 0.3 g$ and $0.5 g$ ) was created with on-board centrifuges. In addition, a simulated microgravity (Random Positioning Machine, RPM) experiment was performed on ground, including both dark-grown and photostimulated samples. Photoactivated samples in spaceflight and RPM experiments showed an increase in the root length consistent with phototropic response to red light, but, as gravity increased, a gradual decrease in this response was observed. Uncoupling of cell growth and proliferation was detected under microgravity in darkness by transcriptomic and microscopic methods, but red light photoactivation produced a significant reversion. In contrast, the combination of red light and partial gravity produced small but consistent variations in the molecular markers of cell growth and proliferation, suggesting an antagonistic effect between light and gravity signals at early plant development. Understanding these parameters of plant growth and development in microgravity will be important as bioregenerative life support systems for the colonization of the Moon and Mars. 


\title{
Keywords
}

Arabidopsis, Cell Growth, Cell Proliferation, Fractional Gravity, Spaceflight, Tropisms.

\author{
Abreviations \\ EMCS European Modular Cultivation System \\ ISS International Space Station \\ RPM Random Positioning Machine \\ SG Seedling Growth \\ WT-Ler Wild type of the Landsberg erecta ecotype of Arabidopsis thaliana
}




\section{Introduction}

Meristematic cells are undifferentiated highly proliferating totipotent cells, which sustain plant development. Ultimately, the basis of plant development is the balance between the cells actively proliferating in the meristems and the cells destined for differentiation (Perrot-Rechenmann 2010). The major functional parameters of meristematic cells are their proliferation rate (i.e. cell division) and their growth rate (i.e. protein synthesis). The strict coordination of these two parameters is called "meristematic competence" (Mizukami 2001). These important parameters are affected by environmental factors including light and gravity, which drive the two major plant tropisms, the directed growth movements by which environmental stimuli modulate plant development (Vandenbrink et al. 2014).

Apart from its direct effect on photosynthesis and phototropism, light is a regulator of plant development through its effects on meristematic cell functions (Griffiths and Halliday 2011; Yoshida et al. 2011). An important group of light receptors in plants are the phytochromes (PhyA to E), a family of proteins differing in the quality of light sensed by each member and the physiological responses they trigger, normally acting in combination. In addition, phytochromes regulate different mechanisms related to plant development by modulating changes in gene expression (Molas and Kiss 2009).

From the earliest developmental stages, light is known to control seed germination and seedling morphogenesis (photomorphogenesis). The morphology of dark-grown (etiolated) and light-grown seedlings is quite different regarding, for example, the hypocotyl length, which is shorter under light irradiation (Jiao et al. 2007). Regarding roots, most reports indicate that illumination shortens roots (Silva-Navas et al. 2015), but other studies show higher rates of root elongation under light irradiation (Laxmi et al. 2008), and, in other cases, no significant differences were observed (BoucheronDubuisson et al. 2016).

In meristems, cell cycle progression is controlled by photoreceptors, but there is increasing evidence of a different effect of light in the root meristems and in the meristems of the aerial part. In the shoot apical meristem, light irradiation activates growth by modulating the levels of the E2F transcription factor family resulting in enhanced rate of cell proliferation (López-Juez et al. 2008). In young seedling plumules, red light irradiation was shown to produce increased mitotic index and nucleolin gene 
expression (Reichler et al. 2001). In contrast, recent data show that light-grown roots al. 2015, 2016). The mechanism of this reduction involves its mediation by flavonols, metabolites whose production is induced by light irradiation (Buer and Muday 2004) and have an effect in the reduction of the root meristematic cell proliferation rate (SilvaNavas et al. 2015, 2016).

Previous experiments under real or simulated microgravity have shown that the signal of the lack of a defined gravity vector is sensed by root meristematic cells in young seedlings, and the result is the disruption of the meristematic competence (Matia et al. 2010). In these cells, this alteration of gravity produces an increase in the cell proliferation rate and a depletion of cell growth, estimated through the rate of ribosome biogenesis, and an increase in the root length (Matía et al. 2010; Manzano et al. 2013; Boucheron-Dubuisson et al. 2016). Similar effects on cell functions have been described in proliferating cells cultured in vitro (Manzano et al. 2016). Specific changes in the percentage of cells in each phase cycle have been found in response to gravity alteration, indicating changes in the regulatory processes of cell cycle progression taking place at cell cycle checkpoints.

There is general agreement in that the phytohormone auxin is the key factor mediating the transduction of the signals from light and gravity receptors to the effect on meristematic cells (Vandenbrink et al. 2014). It has been widely demonstrated that changes in light and gravity stimuli primarily result in alterations in the auxin polar transport. Furthermore, it has been shown that flavonoids, whose synthesis is induced by light and gravity stimuli, have an effect on auxin polar transport regulation and auxin accumulation in the root tip (Buer and Muday 2004).

In this context, we have conducted experiments in real and simulated microgravity aimed to understand how gravity and light responses influence each other and how the combined influence of light and gravity shape plant development. Specifically, we have focused on root development and the alterations found in the root meristematic cell growth and proliferation. For real microgravity and fractional gravity experiments, seedlings have been grown in the International Space Station (ISS); for simulated microgravity experiments, we have used specific well-characterized devices (Herranz et al. 2013), such as the Random Positioning Machine (RPM). Our results show that red 
light is able to compensate for most deleterious effects on the balance of root cell growth and cell proliferation in microgravity.

\section{Materials and methods}

\section{Plant materials and the spaceflight experiment}

In the space experiment Seedling Growth (SG), seeds of the Landsberg erecta ecotype of Arabidopsis thaliana (WT-Ler) as well as of phytochrome mutants phyA-201 and phyB-1 (Kiss et al. 2003) were uploaded to the International Space Station (ISS). Seeds were hydrated and germinated in the European Modular Cultivation System (EMCS) using the culture chambers (cassettes) previously developed for the "Tropi" experiments (Correll et al. 2005). After germination, all seedlings grew in these cassettes for 4 days under continuous white light illumination supplied by an array of LED lights placed in one of the walls of the cassette and $1 g$ gravity, provided by in the EMCS centrifuge, with the gravity vector pointing to the opposite direction of the white light source (Kiss et al. 2012; Vandenbrink et al. 2016). Then, seedlings grew for two additional days at the $g$-level of interest in six runs. Four of these runs, nominally providing $\mu g\left(\leq 10^{-4} \mathrm{~g}\right.$ on ISS), $0.1 g, 0.3 g$, and $1 g$ (in flight-control) were carried out in the SG1 mission, flown in March-May 2013. The two last runs, nominally providing $0.5 g$ and $1 g$ (in flight-control) took place in the SG2 mission, flown in November-December 2014. In all cases, the $g$ vector was obtained by the EMCS centrifuge, and the growth of seedlings occurred under continuous red light illumination supplied by a unilateral light source (Fig. 1). Throughout all steps of the experiments, images were taken from samples and downlinked to the Earth for the analysis of tropisms and morphometric parameters (Vandenbrink et al. 2016). At the end of the 6-day growth period, cassettes were immediately removed from the EMCS and frozen at $-80^{\circ} \mathrm{C}$ in the MELFI freezer on the ISS. In this state, samples were downloaded to the Earth at $-80^{\circ} \mathrm{C}$ in the SpaceX capsule and retrieved in our laboratory for transcriptomic analysis.

\section{On-ground reduced gravity experiment design using the RPM}

In order to validate real microgravity findings (under photostimulation) and provide a comparison with previous experiments on ISS (in dark conditions), a complementary 
experiment on simulated microgravity $\left(\leq 10^{-4} \mathrm{~g}\right.$ on RPM sample area), in a Random (Borst 2009) was performed using the same phytochrome mutants and WT-Ler. It consisted of growing seedlings for 4 days under white light photoperiod (16 h light / $8 \mathrm{~h}$ darkness), followed by 2 days under either continuous red light or darkness (Fig. 2). RPM experimental hardware was provided to ensure similar illumination conditions to that provided by the TROPI hardware on the EMCS (Millar et al. 2010; Kiss et al. 2012); namely the distance from LEDs to seedlings was approx. 40mm, photosynthetic photon flux were $40 \mu \mathrm{mol} \mathrm{m}{ }^{-2} \mathrm{~s}^{-1}$ for White X4 Ultra Bright LED Lamps Reference AND420HWA X6 and $20 \mu \mathrm{mol} \mathrm{m}^{-2} \mathrm{~s}^{-1}$ for Red LED Lamps Reference AND155CRP (Purdy Electronics Corporation). The gravity conditions (either simulated microgravity, or control $1 \mathrm{~g}$ ) were constant throughout the entire growth period of 6 days. Images of seedlings were taken at the end of the experiment. Then, a part of the samples were fixed for $2 \mathrm{~h}$ in $1.5 \%(\mathrm{v} / \mathrm{v})$ glutaraldehyde or $4 \%(\mathrm{v} / \mathrm{v})$ paraformaldehyde in PBS for microscopical observation, and the other part were frozen at $-80^{\circ} \mathrm{C}$ for transcriptomic analysis.

\section{Sample processing for microscopy analyses}

Seedlings fixed in aldehydes were processed for microscopical observation. Samples fixed in 4\% (v/v) paraformaldehyde were washed in PBS, $\mathrm{pH} 7.2$, and treated $10 \mathrm{~min}$ at $37^{\circ} \mathrm{C}$ with a mixture of enzymes for digestion of the cell wall $[2 \%(\mathrm{p} / \mathrm{v})$ celullase (Serva \#16419), $1 \%$ (p/v) pectinase (Sigma \#P4716-25KU), 0.05\% (p/v) Macerozime (Serva \#28302), 0.4\% (p/v) mannitol (Merck \#105983), in PBS containing 10\% (v/v) glycerol and $0.2 \%(\mathrm{v} / \mathrm{v})$ Triton X-100]. Then, root tips were washed twice in PBS, excised and mounted on polylysine-coated multi-well slides, dehydrated in cold methanol (at least $30 \mathrm{~min}$ at $-20^{\circ} \mathrm{C}$ ) and air-dried. Immunofluorescence experiments were performed for detection of the nucleolar protein fibrillarin using mouse monoclonal anti-fibrillarin 38F3 antibody (Abcam \#ab4566, dilution 1:1000) and secondary rabbit anti-mouse IgGAlexa 647nm (Molecular Probes A-21235, dilution 1:100), which were quantitatively assessed for estimation of the nucleolar area.

Samples fixed with 1.5\% (v/v) glutaraldehyde were washed in PBS pH 7.2 for $2 \mathrm{~h}$ at RT, the root tip was excised, dehydrated with ethanol and embedded in Epon as recommended by the manufacturer (four components were mixed EMBED812 
EMS\#14900, Araldite GY 502 EMS\#10900, DDSA EMS\#13710 and DMP30

EMS\#3013600 during $2 \mathrm{~h}$ with gentle shaking, and samples were progressively embedded in 1:2, 1:1 and 2:1 Epon mix: ethanol solutions for $1 \mathrm{~h}$ each at RT, and polymerized on pure Epon mix at $60^{\circ} \mathrm{C}$ for 2 days). Semi-thin sections ( $2 \mu \mathrm{m}$ thick) were observed unstained by phase contrast microscopy (Leica DM2500 microscope with a CCD Leica DFC320 camera) and the differences in cell proliferation rate were estimated by counting the number of cells per millimeter in the cellular rows corresponding to future epidermis and cortex of the root meristem in each condition.

\section{Sample processing for transcriptional analyses}

Frozen samples were dissected and only the roots were processed for transcriptomic analysis with micropestles (Sigma \#Z317314) using a plant specific RNA extraction column kit (Norgen Bioteck \#48400). RNA quality was determined by Nanodrop2000 (Thermo) assays after DNAse-turbo (AmbioLife\#1907) treatment. Quantitative RTPCR (qPCR) was performed in a single step with a IQ5 Real Time Detection System (Biorad) with probes corresponding to selected genes involved in auxin polar transport, cell cycle regulation, and ribosome biogenesis (Suppl. Table S1). The nucleic acid stain used was SYBR green (Agilent Technologies \#600886). RT-qPCR were performed in standardized conditions ( 35 cycles and $60^{\circ} \mathrm{C}$ hybridization phase temperature) after two melting curve quality tests of the primer pairs. First, we tested that all probes combinations produced a single peak (amplicon) and second, the RNA/probe concentration ratios were in the optimal efficiency range of the qPCR reaction. The data were analyzed with IQ5 Optical System Software (Biorad) and processed with Microsoft Excel (Microsoft Office 2007).

\section{Results}

\section{Seedling Growth space experiments (micro-g and fractional-g effect on photoactivated samples)}

We determined the root length from images of the Seedling Growth-1 (SG1) experiment obtained by on-flight image (Vandenbrink et al. 2016) at the sixth day of growth (Fig. 1). The measurement of the root length showed a general increase in this parameter in 
all samples grown in micro- $g$. This increase was significant in all strains, WT-Ler, phyA and phyB mutant samples (Fig. 2a). In addition to the samples exposed to ISS microgravity environment, the SG experiment included the exposure of a similar set of samples to centrifugation to generate nominal $0.1 g, 0.3 g$ and $0.5 g$ conditions. Root length showed a general increase in all partial gravity samples, but the intensity of the effect was reduced as the gravitational load increased, being conspicuous (although not significant for phyA) at $0.1 \mathrm{~g}$, remaining significant only in WT-Ler seedlings at $0.3 g$ and only appearing as a small reduction trend in the $0.5 \mathrm{~g}$ samples, irrespective of the strain used (Fig. 3). The roots of $p h y B$ mutant samples were longer than those of WTLer in all cases. This root elongation as a consequence of microgravity is in agreement with previous results in our spaceflight experiments (Kiss et al. 2012; Vandenbrink et al. 2016).

In a transcriptomic study, we included qPCR experiments performed from root RNA extracted from the SG1 and SG2 samples that were frozen on-board and recovered from the ISS. The analysis revealed the differences in the expression of three sets of genes, selected as markers of different functions. The first set was composed by two genes involved in auxin polar transport and perception (PIN-Formed 2, PIN2 and Transport Inhibitor Response 1 TIRl); the second set was composed by genes involved in cell cycle regulation (Casein Kinase $2 \mathrm{~A} C K 2 \mathrm{~A}$ and Cyclin B1, CYCB1), indicative of cell proliferation; finally, the third set was composed by genes involved in the regulation of ribosome biogenesis (fibrillarin, $F I B$, and nucleolin 1, NUC1), indicative of cell growth (Fig. 4). Interestingly, the pattern of gene expression obtained in microgravity and partial gravity conditions was quite different. Under microgravity, in WT-Ler and phyB mutant, all gene markers were upregulated when compared to $1 g$ control. Markers of auxin polar transport, cell cycle progression and ribosome biogenesis showed an enhanced expression in microgravity, especially for CYCB1 in WT-Ler. The enhancement was more significant for WT-Ler than for $p h y B$. In contrast, phyA mutant, showed no significant variations in all the analyzed genes, with a slight decrease in expression in the cell growth marker genes (Fig. 4a).

Under partial gravity (Fig. 4b-d), the WT-Ler samples showed very small and nonsignificant alterations, except in the auxin polar transport genes. In contrast to the microgravity results, both $p h y A$ and phyB samples showed a similar trend under partial $g$, with significant decrease in the expression of at least one of the genes used to 
evaluate cell growth, cell proliferation and auxin polar transport. Noteworthy, those differences were proportional to the $g$-load, but always remaining relatively small (barely reaching a 2-fold decrease in the most variable sample). The samples exposed to $0.1 \mathrm{~g}$ showed small variations, some of them statistically significant, which became more consistent and quantitatively much more evident in the $0.3 g$ and $0.5 g$ samples. A decrease in the expression of both nucleolar marker genes in the phyA and phyB mutants, but not in the wild type, and in the PIN2 in all strains, was clearly observed.

\section{RPM simulated microgravity experiment (red light photostimulation effect)}

With the purpose of extending the information obtained from the spaceflight experiments, including a broader range of methodological approaches, a complementary experiment was carried out on ground, using simulated microgravity (desktop RPM 2.0 in real random mode). We used the same set of genotypes as in the space experiment, and similar illumination conditions as described in the material and methods section. The main difference with the space experiment was during the first four days of growth, when seedlings grew under simulated microgravity, combined with a $16 \mathrm{~h} / 8 \mathrm{~h}$ photoperiod of white light illumination, whereas in space, growth during this period was at $1 g$ under continuous white light. These conditions allow doing two complementary experiments: (i) The goal of the ISS experiment was to characterize the response of seedlings when they are exposed simultaneously to the two factors implicated: microgravity and red light. Thus, seedlings grew firstly with the same conditions (4 days under $1 g$, continuous light) in order to obtain a homogeneity population before exposure to migro- $g$ or fractional- $g$ and red light or darkness. (ii) The goal of the experiment performed on-ground was to characterize the response of the plants to the red light once they have already responded to the microgravity. Thus, these conditions allow analyzing the response of the plant to a supplementary factor. In this experiment, we chose the environmental factors that allow plant metabolism as close as possible to the physiological conditions: 4 days of photoperiod of $16 \mathrm{~h} / 8 \mathrm{~h}$ with white light.

In both cases, spaceflight and RPM, the conditions of the last two days included experimental gravity levels and either continuous red light, or darkness (Fig. 2). A control $1 g$ experiment, applying the same parameters of illumination, was run in parallel. 
This experimental setup allowed us to perform a preliminary test on whether or not the

mutant line and the illumination conditions could produce any effect per se on the parameters under study, irrespective of the gravity alteration. The test was performed on the samples grown under $1 g$ gravity control conditions, by comparing samples grown under different illumination conditions, and we measured the root length, the proliferation rate and the size of the nucleolus, a parameter indicative of cell growth, in root meristematic cells. Our initial analysis, showed very small changes in root length (Fig. 2). However, when quantified, roots were longer in the phyB mutant than in WTLer, reaching statistical significance only in non-photoactivated conditions (Fig. 5). Red-light photoactivated seedlings were consistently longer than non-photoactivated ones, even though statistical significance was only reached in the phyA mutant (Fig. 5). Cell proliferation rate was determined in the three cellular layers of the root meristem, from microscopy preparations of fixed samples (Suppl. Fig. S1). The result was that the cell proliferation rate in the root meristem was significantly enhanced by red light in WT-Ler and in phyB mutant (Fig. 6). In turn, cell growth rate was estimated in the same root meristematic cells by measuring the nucleolar size as detected by immunofluorescence studies with anti-fibrillarin antibodies (Suppl. Fig. S2), resulting in the detection of a similar enhancement in this parameter (Fig. 7). Therefore, red light illumination itself appeared to have an enhancing effect on these essential root developmental parameters of meristematic cells, except in the case of phyA.

\section{RPM simulated microgravity experiment (microgravity effect)}

When comparing the simulated microgravity samples with the corresponding $1 g$ control, we found that seedlings were more disoriented to one another, as expected by the RPM randomization of the gravity vector. The longer and continuous simulated microgravity condition throughout the entire period of growth, from germination, can account for this feature, although the orientation of the seedlings is maintained due to the spatial reference of the source of light (Fig. 2). The simulated microgravity effects on WT-Ler and the phyB mutant were quite similar to each other and they showed marked differences with the results of the phyA mutant, consistently with the spaceflight SG1 data. Thus, simulated microgravity increased the root length on seedlings not photoactivated with red light of WT-Ler and phyB mutant, but not in the phyA mutant, 
and the red light treatment reversed this effect, producing roots of similar length, irrespective of the genotype and of the gravity condition (Fig. 8).

Regarding the proliferation rate, simulated microgravity enhanced cell proliferation in WT-Ler and $p h y B$ in comparison to the $1 g$ control, and red light reversed this effect, even producing a decrease in this parameter (Fig. 9). The phyA mutant showed the opposite behavior, with a decrease of the cell proliferation rate under simulated microgravity, which was suppressed by red light irradiation (Fig. 9). The increase of the proliferation rate in WT-Ler and $p h y B$ mutant exposed to simulated microgravity, but not to red light photoactivation, was in agreement with the observed trend to overexpression of CYCB1 cell cycle regulation gene in these lines without photoactivation (Fig. 10). In contrast, the phyA mutant showed a significant depletion in the expression of the cell cycle genes (Fig. 10a). In general, the effects of simulated microgravity at the level of gene expression were completely reverted by red light photostimulation (Fig. 10b).

In terms of cell growth rate (estimated by the nucleolar size), a general trend was detected under simulated microgravity, consisting of the decrease of this parameter in all non-photostimulated samples. This decrease reached statistical significance in both phyA and phyB mutants (Fig. 11). Red light treatment did not alter this decrease except in the case of the phyA mutant and, interestingly, the WT-Ler showed the most intense effect in the simulated microgravity treatment (Fig. 11). These general inhibiting effects, estimated by microscopical methods, were in agreement with the downregulation of nucleolin and fibrillarin genes observed without red light photostimulation (particularly significant in phyB mutant, Fig. 10a). However, in terms of gene expression, the red light treatment was able to recover fibrillarin and nucleolin transcriptional levels (Fig. 10b).

\section{Discussion}

Our results involving microgravity (real or simulated), or partial $g$ conditions (SG1/SG2), in combination with red light photostimulation, show complex responses in plants, particularly regarding the differential effects observed in the phytochrome mutants exposed to several low-gravity conditions. Given that the experiments involved a photoactivation treatment with red light during the two last days of seedling growth, it 
is worth noting that both $p h y A$ and $p h y B$ mutants have a compromised perception of the expected under the combined light and gravity conditions that have been tested (Kiss et al. 2003), especially taking into account that phytochrome $\mathrm{B}$ is the main receptor specialized in red light sensing and perception under $1 g$ conditions (Molas and Kiss 2009).

\section{Red light photoactivation compensates for the deleterious effects on microgravity on cell growth and proliferation}

Taking together, our results show a positive effect of the red-light photoactivation treatment in the compensation of the alterations induced by the microgravity environment on cell growth and proliferation. As shown by our laboratories in previous papers (Matía et al. 2010; Manzano et al. 2013; Boucheron-Dubuisson et al. 2016), the main alteration observed in root meristematic cells grown in a microgravity environment is the disruption of meristematic competence, that is, the coordination of cell proliferation and cell growth (Mizukami 2001). Whereas under microgravity, the cell proliferation rate is enhanced and the cell growth rate is inhibited, photoactivation by red light appears to restore the coordination, although important differences can be observed in different experimental conditions. In Table 1, we summarize the effects of the different environmental parameters (illumination/gravitational conditions) for each one of the genotypes tested (WT-Ler and the phyA and phyB mutants).

The results of the space experiment, incorporating red light photoactivation, showed enhanced expression of marker genes of cell cycle regulation and ribosome biogenesis in the WT-Ler and $p h y B$ under SG1 microgravity conditions. The enhancement was more significant for the WT-Ler than for phyB. Taken together, the results on the factors related to cell proliferation and cell growth show a concerted expression of these two sets of genes, which corresponds to the normal physiological pattern of meristematic cells, and should be more likely due to the red light photoactivation.

Furthermore, red light shows a general positive effect on the transduction of the light and gravity signals mediated by auxin (Medina and Herranz 2010) by inducing the normalization of the auxin polar transport, which is affected under microgravity conditions (Herranz et al. 2014). Also in this case, there are experimental differences depending on particular conditions. 
Under simulated microgravity, the results showed a similar trend as reported in previous proliferation in the root meristem, and a partial reversion of these effects by the red light photoactivation, which occurs mainly through cell proliferation, but not cell growth as shown in our data. The result was that, after red light irradiation, both parameters appeared reduced in comparison to the values obtained in control $1 g$ gravity. Since both microgravity (lack of gravitropic stimulus) and red light irradiation may be increasing the cell proliferation rate through similar pathways (e.g. those involving CK2 or PIF activity (Herranz et al. 2014)), the absence of gravity should have minimal effects if the pathway is already activated by red light. The phytohormone auxin is the key factor mediating transduction of the signals from light and gravity receptors to the effect on meristematic cells (Vandenbrink et al. 2014). In turn, flavonoids, whose synthesis is also induced by light and gravity stimuli, have an effect on auxin polar transport regulation and auxin accumulation in the root tip (Buer and Muday 2004). It is also known that auxin controls both cell cycle progression (through cyclinA-B and CDK-A gene expression) and cell elongation. Considering all these facts, we assume that the concerted action of the gravitropic and light stimuli could lead to the modification of the auxin polar transport and, consequently, to the re-distribution of this hormone. The effect of such a new distribution on the root meristem would be the induction of alterations in cell growth and proliferation, allowing a partial reversion of microgravity effects by red light photoactivation.

However, in terms of meristematic competence, our results suggest that red light irradiation restored the concert of the two parameters that define this feature. Actually, we have found different cases in which meristematic competence (coordination of cell growth and proliferation) is restored after its disruption caused by microgravity. We have defined in Table 1 with the term "fast" the case of enhancement of both parameters and upregulation of the marker genes, and with the term "slow" the situation of concerted reduction of the rates of both parameters and downregulation of the marker genes. In the case of "slow" meristematic competence, cells are indeed "competent" but they show a depleted level of activity (i.e. decelerated growth and proliferation).

An additional clarification of the role played by red light in the regulation of the functions of meristematic cells was obtained from the comparison of the $1 g$ control runs 
of the RPM experiment, with and without the red light photoactivation pulse. These experiments are in agreement with the interpretation of the results of the space experiment, suggesting that red light irradiation is indeed a positive factor for restoration of meristematic competence disrupted by microgravity.

\section{Differential microgravity responses in the two phytochrome mutants}

While the results of $p h y B$ are very similar to WT-Ler under microgravity, phyA seems to be much more resilient to the absence of the gravity vector, showing quite normal meristematic competence parameters despite a longer root length is detected in these conditions. In fact, the phyA mutant keeps meristematic competence either in real or simulated microgravity in the dark, but showing reduced rates of cell proliferation and cell growth ("slow" meristematic competence). Both of these rates are increased by red light photoactivation, reaching values slightly higher than those reached by the in-flight or in-ground $1 g$ controls. These results support once again that there is a contribution of the light/phototropic signal to the cell proliferation regulation, which can counteract some of the deleterious effects of the loss of gravitational signal in darkness. In turn, the phyB mutant is able to recover the meristematic competence, which was lost in real or simulated microgravity, as an effect of the photoactivation pulse with red light. This recovery is of the "fast" mode (i.e., the same as the WT-Ler, Fig. 4, Table 1).

The magnitude of the changes induced by red light photoactivation in the phyA mutant is not statistically significant in any real or simulated microgravity experiment. Therefore, these changes should be only taken as general trends, better than real alterations severely affecting functional processes. Otherwise, if we analyze the effects of the genetic mutation irrespective of the environmental conditions, the phyA mutant shows a lower cell proliferation rate and higher cell growth activity compared to WTLer.

A higher activity of auxin polar transport is also observed in photoactivated WT-Ler and phyB mutant, but not in the phyA mutant. This observation is an additional indication that the link between the phototropic signal sensed by phyA and auxin polar transport and perception may be important to restore the functional balance that gives account of meristematic competence.

It is important to note that these effects of light and altered gravity on the WT-Ler and phytochrome mutants are accompanied by an altered phototropic behavior of these 
strains under spaceflight conditions, as we have found from our previous spaceflight experiments (Millar et al. 2010; Kiss et al. 2012). In fact, all strains show a number of phototropic responses in space that are not observable under $1 g$ conditions; particularly, the roots show a positive red-light phototropic response inversely correlated to the magnitude of the gravity vector (Vandenbrink et al. 2016).

\section{Differential gravitational responses at partial-g levels}

We have found a decrease in both cell growth and cell proliferation rates (not leading to meristematic competence alteration but just to a "slower" activity level) at the $0.3 g-0.5 g$ level. These observed effects occur in the two phytochrome mutants, but not in the WTLer (see Table 1), suggesting that an optimal perception of the red stimulus could be enough to compensate the alterations related to the partial gravity environments. These observations depict an increasingly complex scenario for the plants, in which both a stimulating phototropic signal and a $0.3-0.5 \mathrm{~g}$ load are pulling in opposite directions the developmental pattern of the cell. In this case, the auxin polar transport is decreased by the increasing partial gravity signal, and this effect also occurs in the wild type. It was shown that statoliths could still be sensing gravity in the root tips at $0.1 g-0.5 g$, apparently triggering a normal gravitropism signal (Perbal and Driss-Ecole 1994; Kiss 2014).

\section{The importance of simulated microgravity experiments in ground-based facilities}

This work also emphasizes the advantages of complementing space experiments performed in real microgravity with parallel experiment in ground-based simulated microgravity facilities (Kraft et al. 2000; Herranz et al. 2013; Boucheron-Dubuisson et al. 2016). The results obtained in real microgravity (ISS experiment) were clearer, showing greater differences between experimental and control samples. However, ground-based facilities provided us with the possibility of testing more parameters and conditions, providing supporting data of high interest. In addition, the cross-comparison of real and simulated microgravity experiments allows improving the simulation technologies on ground. Further experimentation in partial $g$ conditions should be considered in the near future, since the alterations observed in both the phototropic response (Vandenbrink et al. 2016) and the cell growth/cell proliferation balance may 
be important to define the Mars-related settings for plant breeding below the Earthnominal gravity level (Kiss 2014). In fact, particular mutants, as phyA, less sensible to the meristematic competence lost under low gravity and light conditions, could be good candidates to be added into extraterrestrial life support systems. Nevertheless, even considering the different effects observed under real or simulated microgravity, and even the partial gravity levels in the range close to Moon and Mars surface, most of parameters disturbanced were affected in a similar manner. Red light photoactivation is able to coordinate the rates of cell proliferation and cell growth, and, thus, red light causes the recovery of the meristematic competence after gravitational stress.

\section{Supplementary material online}

Two figures and one table have been included as supplementary material to this article.

\section{Author contribution statement}

All authors participated in the design of the experiments and in the preparation of the spaceflight project, except VPL, who provided the simulated microgravity facility. MAV, AM and JPV prepared and processed the spaceflight and RPM seedling samples. JZK, RH and FJM wrote the manuscript. All authors read and approved the manuscript.

\section{Acknowledgments}

Funding for this study was provided mainly by the Spanish National Plan for Research and Development (MINECO-ERDF co-funding) Grant ESP2015-64323-R to FJM. The access to ISS and RPM facilities was granted by ESA-ELIPS ILSRA-2009-0932 to FJM and GBF Program GIA Project (contract\# 4000105761) to RH. This research was supported also by grants (NNX12A0656 and 80NSSC17K0546) from NASA to JZK and the French Space Agency- CNES to ECD and VPL. MAV and AM were recipients of grants of the Spanish National Program for Young Researchers Training (Refs. BES2010-035741 and BES-2013-063933, respectively). We would like to thank the skillful technical assistance of Mrs. Mercedes Carnota (CIB-CSIC), the fine support of NASA's 
Ames Research Center in the use of TROPI hardware, and the European Space Agency support throughout the entire "Seedling Growth" space project. Finally, we would like to thank the astronauts on board the ISS for their work, without whom these experiments would have not been possible.

\section{References}

Borst A, van Loon JJ (2009) Technology and development for the Random Positioning Machine, RPM. Microgravity Sci Technol 21: 287-292

Boucheron-Dubuisson E, Manzano AI, Le Disquet I, Matia I, Saez-Vasquez J, van Loon JJ, Herranz R, Carnero-Diaz E, Medina FJ (2016) Functional alterations of root meristematic cells of Arabidopsis thaliana induced by a simulated microgravity environment. J Plant Physiol 207: 30-41

Buer CS, Muday GK (2004) The transparent testa4 mutation prevents flavonoid synthesis and alters auxin transport and the response of Arabidopsis roots to gravity and light. Plant Cell 16: 1191-1205

Correll MJ, Edelmann RE, Hangarter RP, Mullen JL, Kiss JZ (2005) Ground-based studies of tropisms in hardware developed for the European Modular Cultivation System (EMCS). Adv Space Res 36: 1203-1210

Griffiths J, Halliday K (2011) Plant development: light exposure directs meristem fate. Curr Biol 21: R817-819

Herranz R, Anken R, Boonstra J, Braun M, Christianen PCM, Geest Md, Hauslage J, Hilbig R, Hill RJA, Lebert M, Medina FJ, Vagt N, Ullrich O, Loon JJWAv, Hemmersbach R (2013) Ground-based facilities for simulation of microgravity, including terminology and organism-specific recommendations for their use. Astrobiology 13: 1-17

Herranz R, Valbuena MA, Youssef K, Medina FJ (2014) Mechanisms of disruption of meristematic competence by microgravity in Arabidopsis seedlings. Plant Signal Behav 9: e28289

Jiao Y, Lau OS, Deng XW (2007) Light-regulated transcriptional networks in higher plants. Nat Rev Genet 8: 217-230

Kiss JZ (2014) Plant biology in reduced gravity on the Moon and Mars. Plant Biol (Stuttg) 16 Suppl 1: 12-17

Kiss JZ, Millar KD, Edelmann RE (2012) Phototropism of Arabidopsis thaliana in microgravity and fractional gravity on the International Space Station. Planta 236: 635-645

Kiss JZ, Mullen JL, Correll MJ, Hangarter RP (2003) Phytochromes A and B mediate red-light-induced positive phototropism in roots. Plant Physiol 131: 1411-1417

Kraft TF, van Loon JJ, Kiss JZ (2000) Plastid position in Arabidopsis columella cells is similar in microgravity and on a random-positioning machine. Planta 211: 415422

Laxmi A, Pan J, Morsy M, Chen R (2008) Light plays an essential role in intracellular distribution of auxine carrier PIN2 in Arabidopsis thaliana. PLoS ONE 3: e1510

López-Juez E, Dillon E, Magyar Z, Khan S, Hazeldine S, de Jager SM, Murray JAH, Beemster GTS, Bögre L, Shanahan H (2008) Distinct light-Initiated gene 
expression and cell cycle programs in the shoot apex and cotyledons of Arabidopsis. Plant Cell 20: 947-968

Manzano AI, Herranz R, Manzano A, Van Loon JJWA, Medina FJ (2016) Early effects of altered gravity environments on plant cell growth and cell proliferation: Characterization of morphofunctional nucleolar types in an Arabidopsis cell culture system. Front Astron Space Sci 3

Manzano AI, Larkin O, Dijkstra C, Anthony P, Davey M, Eaves L, Hill R, Herranz R, Medina FJ (2013) Meristematic cell proliferation and ribosome biogenesis are decoupled in diamagnetically levitated Arabidopsis seedlings. BMC Plant Biol 13: 124

Matía I, González-Camacho F, Herranz R, Kiss JZ, Gasset G, van Loon JJWA, Marco R, Medina FJ (2010) Plant cell proliferation and growth are altered by microgravity conditions in spaceflight. J Plant Physiol 167: 184-193

Medina FJ, Herranz R (2010) Microgravity environment uncouples cell growth and cell proliferation in root meristematic cells: The mediator role of auxin. Plant Signal Behav 5: 176-179

Millar KD, Kumar P, Correll MJ, Mullen JL, Hangarter RP, Edelmann RE, Kiss JZ (2010) A novel phototropic response to red light is revealed in microgravity. New Phytol 186: 648-656

Mizukami Y (2001) A matter of size: developmental control of organ size in plants. Curr Opinion Plant Biol 4: 533-539

Molas ML, Kiss JZ (2009) Phototropism and gravitropism in plants. Adv Bot Res 49: 134

Perbal G, Driss-Ecole D (1994) Sensitivity to gravistimulus of lentil seedling roots grown in space during the IML 1 Mission of Spacelab. Physiol Plant 90: 313-318

Perrot-Rechenmann C (2010) Cellular responses to auxin: division versus expansion. Cold Spring Harbor Perspectives in Biology 2: a001446

Reichler SA, Balk J, Brown ME, Woodruff K, Clark GB, Roux SJ (2001) Light differentially regulates cell division and the mRNA abundance of pea nucleolin during de-etiolation. Plant Physiol 125: 339-350

Silva-Navas J, Moreno-Risueno MA, Manzano C, Pallero-Baena M, Navarro-Neila S, Tellez-Robledo B, Garcia-Mina JM, Baigorri R, Gallego FJ, del Pozo JC (2015) D-Root: a system for cultivating plants with the roots in darkness or under different light conditions. Plant J 84: 244-255

Silva-Navas J, Moreno-Risueno MA, Manzano C, Tellez-Robledo B, Navarro-Neila S, Carrasco V, Pollmann S, Gallego FJ, Del Pozo JC (2016) Flavonols mediate root phototropism and growth through regulation of proliferation-to-differentiation transition. Plant Cell 28: 1372-1387

Vandenbrink JP, Herranz R, Medina FJ, Edelmann RE, Kiss JZ (2016) A novel bluelight phototropic response is revealed in roots of Arabidopsis thaliana in microgravity. Planta 244: 1201-1215

Vandenbrink JP, Kiss JZ, Herranz R, Medina FJ (2014) Light and gravity signals synergize in modulating plant development. Front Plant Sci 5: 563

Yoshida S, Mandel T, Kuhlemeier C (2011) Stem cell activation by light guides plant organogenesis. Genes Dev 25: 1439-1450 
Fig. 1 Images of 6-day-old seedlings from SG1 and SG2 experiments performed on the International Space Station. One culture chamber (cassette) per experimental condition is shown, taken at the end of the experiment (6 days after seed hydration). Seedlings of the different genotypes (WT-Ler, phyA and phyB mutants) were grown either under microgravity $(\mu \mathrm{g})$, partial gravity $(0.1 g, 0.3 g$ or $0.5 g)$ or $1 g^{\text {FLIGHT }}$ control conditions. All samples were exposed to continuous white light for 4 days, from top of the cassette and with continuous red light (photoactivation) for the last two days, with the light source located laterally (left), as shown in the figure.

Fig. 2 Images of 6-day-old seedlings from the RPM experiment. a Samples with no photoactivation. b Samples with red light photoactivation the last 2 days of the experiment. The same genotypes used in the ISS experiment were exposed to simulated microgravity (RPM) or $1 g$ control conditions. Seedlings were grown for six days after seed hydration, the first four days under light photoperiod, and the last two days seedlings were either subjected to red light photoactivation or remained in darkness. Scale bar indicates $3 \mathrm{~mm}$.

Fig. 3 Length of the primary root in seedlings grown in the ISS (SG1 and SG2 experiments) at $\mu g$ (a), $0.1 g$ (b), $0.3 g$ (c), $0.5 g$ (d) next to the corresponding $1 g^{\text {FLIGHT }}$ control. Bars indicate standard deviation. Asterisks indicate significant difference ( $\mathrm{t}$ test, $P<0.05)$ between samples of the same genotype exposed to $1 g^{F L I G H T}$ gravity conditions (EMCS centrifuge).

Fig. 4 Relative gene expression estimated by quantitative PCR (qPCR) in samples of the SG1/SG2 space experiment [nominally $0 g(\mu g), 0.1 g, 0.3 g$ and $0.5 g$ ]. Three sets of genes have been analyzed, which were two genes of the auxin polar transport perception (EIR1, also known as PIN2, and TIR1), two genes of the cell cycle regulation (CK2A and $C Y C B 1)$ and two genes involved in the regulation of ribosome biogenesis ( $F I B$ and $N U C 1)$. Histograms indicate the difference in expression $(\log 2$ Ratio between the same samples grown in $\mu g$ (a), $0.1 g$ (b), $0.3 g$ (c), $0.5 g$ (d) versus $1 g^{F L I G H T}$ control). Asterisks indicate significant values ( $\mathrm{t}$ test, $P<0.05$ ). 
Fig. 5 Primary root length in seedlings grown under control $1 g$ conditions, in the Asterisks indicate significant difference $(\mathrm{t}$-test, $P<0.05)$ between samples of the same genotype in different illumination conditions. The symbol (\#) indicates significant difference (t-test, $P<0.05$ ) with respect to WT-Ler under the same illumination regime.

Fig. 6 Cell proliferation rate in root meristematic cells of seedlings grown under control $1 g$ conditions, in the context of the simulated microgravity experiment. Bars indicate standard deviation. Asterisks indicate significant difference (t-test, $P<0.05)$ between samples of the same genotype in different illumination conditions. The symbol (\#) indicates significant difference (t-test, $P<0.05)$ with respect to WT-Ler under the same illumination regime.

Fig. 7 Cell growth rate (estimated by nucleolus cross-sectional area), in root meristematic cells of seedlings grown under control $1 g$ conditions, in the context of the simulated microgravity experiment. Bars indicate standard deviation. Asterisks indicate significant difference (t-test, $P<0.05$ ) between samples of the same genotype in different illumination conditions. The symbol (\#) indicates significant difference (t-test, $P<0.05$ ) with respect to WT-Ler under the same illumination regime.

Fig. 8 Primary root length in seedlings grown under simulated microgravity (RPM) and control $1 g$ conditions. a Samples with no photoactivation. b Samples with red light photoactivation during the last 2 days of the experiment. Bars indicate standard deviation. Asterisks indicate significant difference (t-test, $P<0.05)$ between samples of the same genotype in different gravity conditions.

Fig. 9 Cell proliferation rate in root meristematic cells of seedlings grown under simulated microgravity (RPM) and control $1 g$ conditions. a Samples with no photoactivation. b Samples with red light photoactivation during the last 2 days of the experiment. Bars indicate standard deviation. Asterisks indicate significant difference (t-test, $P<0.05)$ between samples of the same genotype in different gravity conditions. 
Fig. 10 Relative gene expression estimated by quantitative PCR (qPCR) in samples of the RPM experiment. a Samples with no photoactivation. b Samples with red light photoactivation during the last 2 days of the experiment. Three sets of genes have been analyzed, namely two genes of the auxin polar transport and perception (EIRI, also known as PIN2, and TIR1), two genes of the cell cycle regulation (CK2A and CYCB1), and two genes involved in the regulation of ribosome biogenesis (FIB and NUC1). Histograms indicate the difference in expression ( $\log 2$ Ratio between the same samples grown in RPM (simulated $\mu g$ versus $1 g$ control). Asterisks indicate significant values ( $\mathrm{t}$ test, $P<0.05)$.

Fig. 11 Cell growth rate (estimated by nucleolus cross-sectional area), in root meristematic cells of seedlings grown under simulated microgravity (RPM) and control $1 g$ condition. a Samples with no photoactivation. b Samples with red light photoactivation during the last 2 days of the experiment. Bars indicate standard deviation. Asterisks indicate significant difference (t-test, $P<0.05)$ between samples of the same genotype in different gravity conditions. 


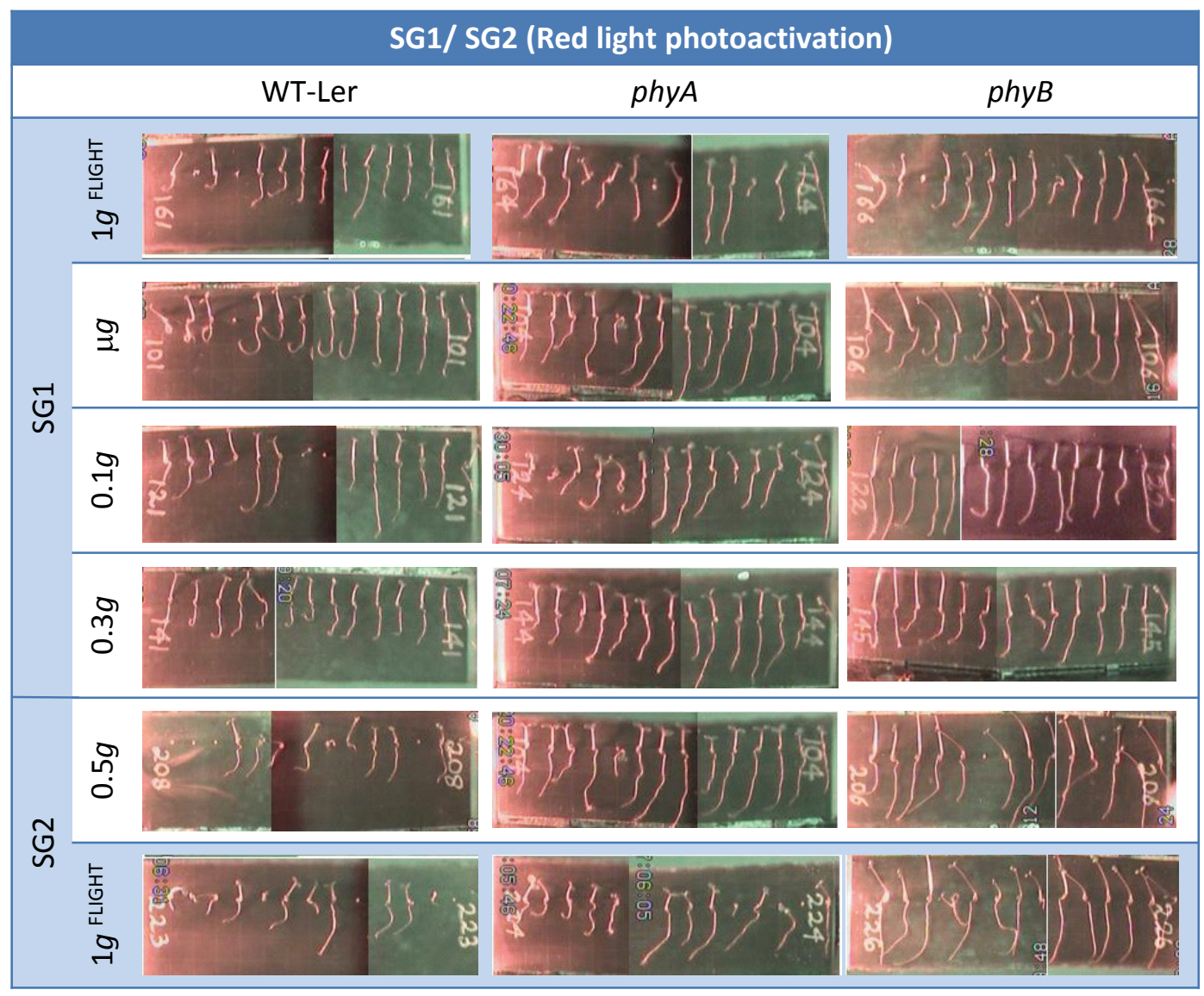




\section{a) No photoactivation}

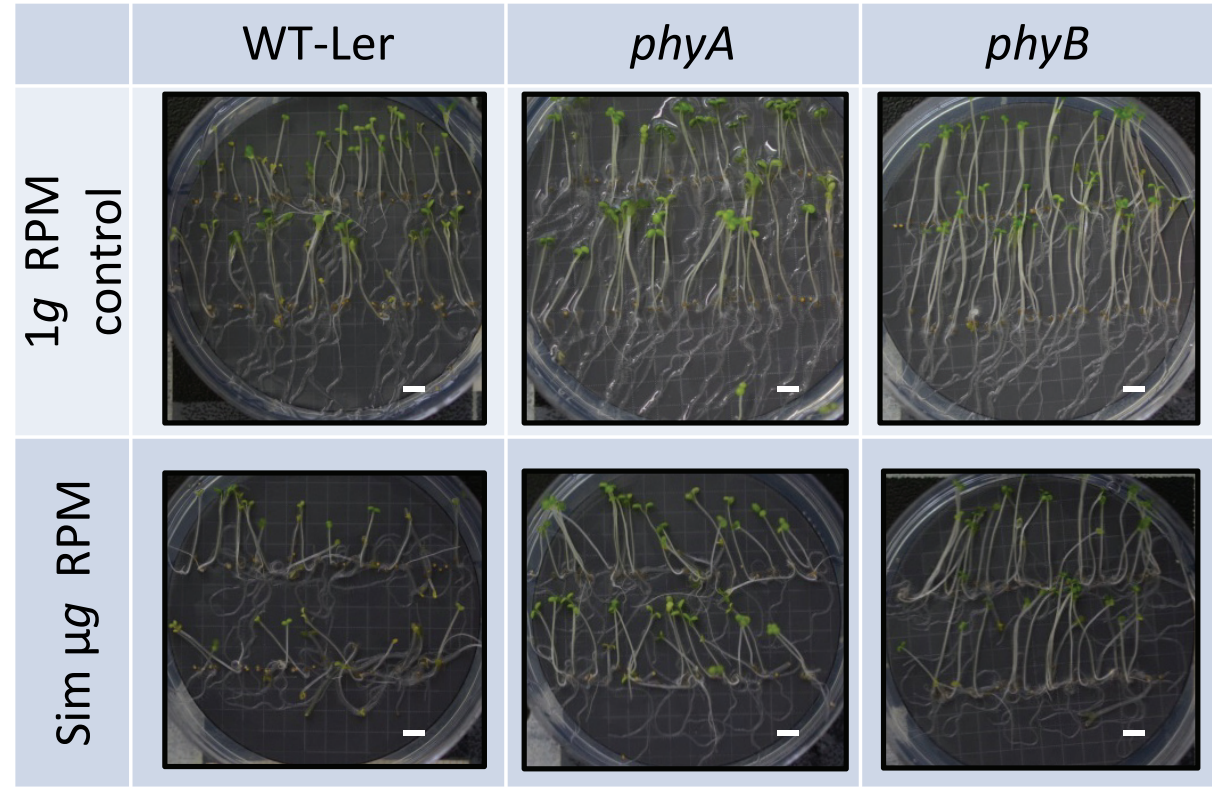

b) Red light photoactivation

WT-Ler

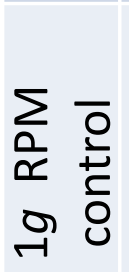

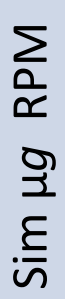

phyA
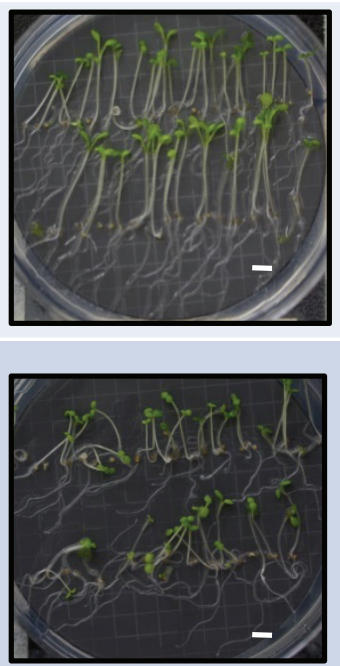

phyB
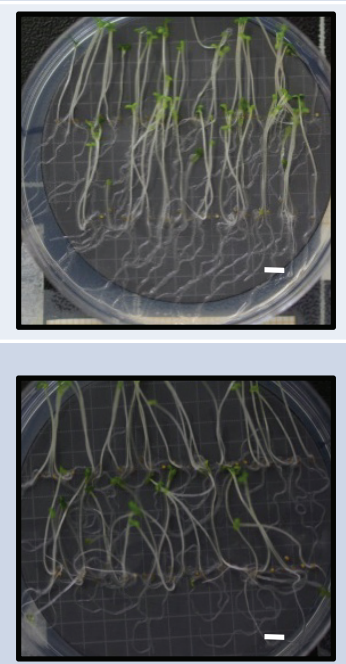
a)

SG1: $\mu g$

(Red light photoactivation)

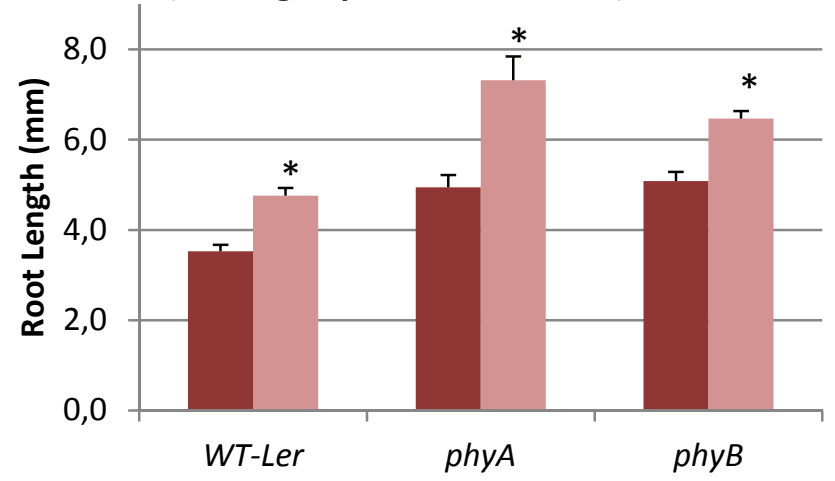

c)

SG1: $0.3 g$

- $1 g^{F L I G H T}$

(Red light photoactivation)

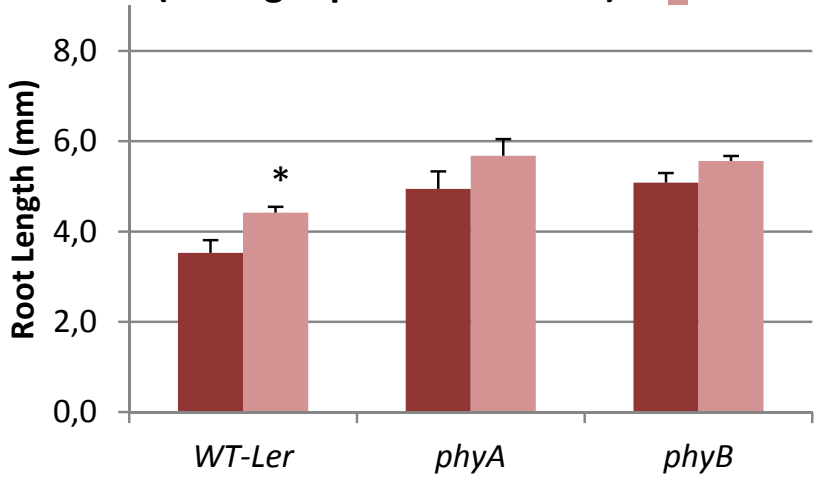

b)

SG1: $0.1 g$

- $1 g^{\text {FLIGHT }}$

(Red light photoactivation) $\quad 0.1 \mathrm{~g}$

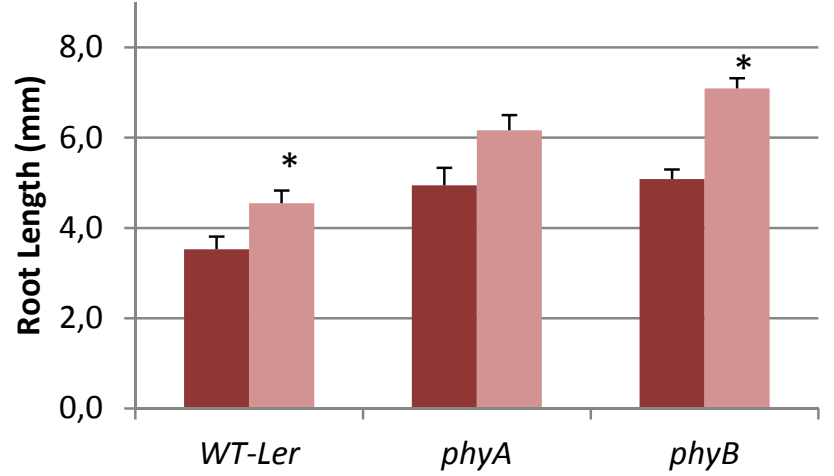

d)

SG2: $0.5 g$

- $1 g^{\text {FLIGHT }}$
- $0.5 g$

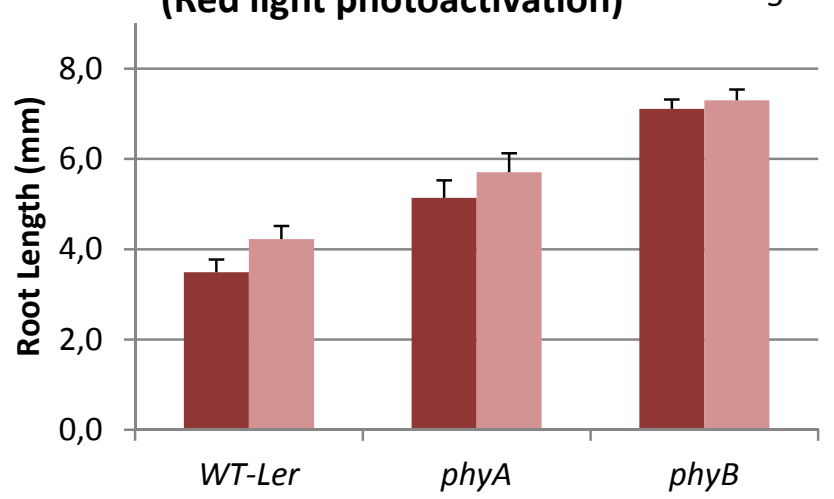




\section{SG1/SG2 (Red light photoactivation)}

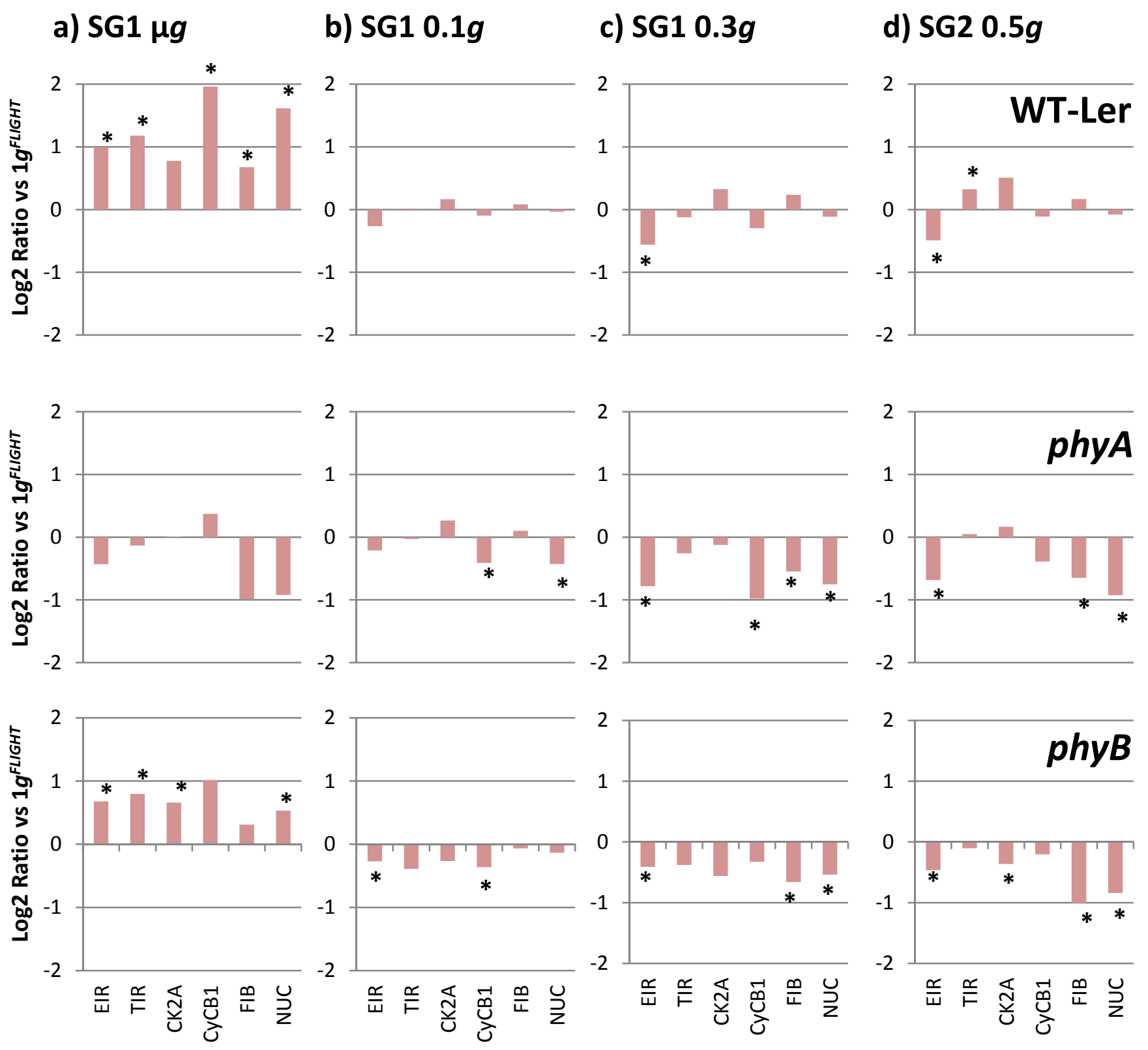




\section{$1 g$ RPM controls}

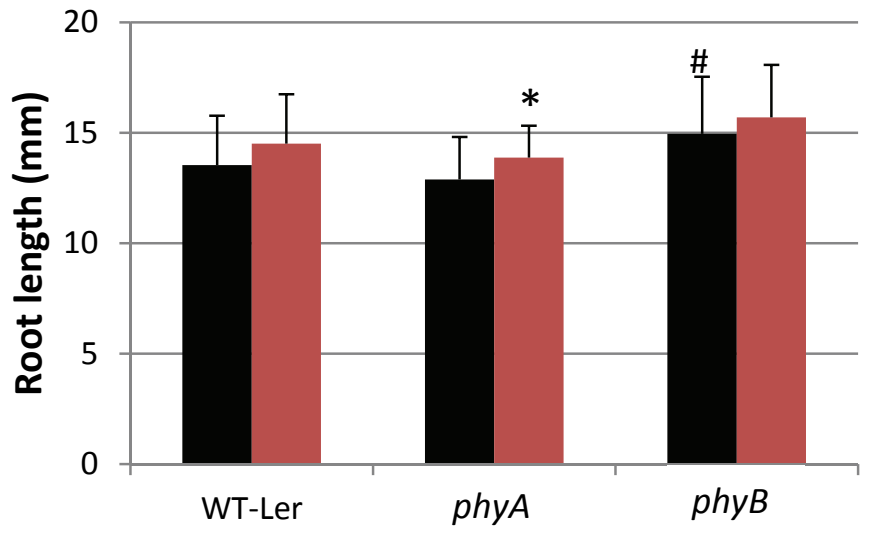

No photoactivation Red light photoactivation 


\section{$1 g$ RPM controls}

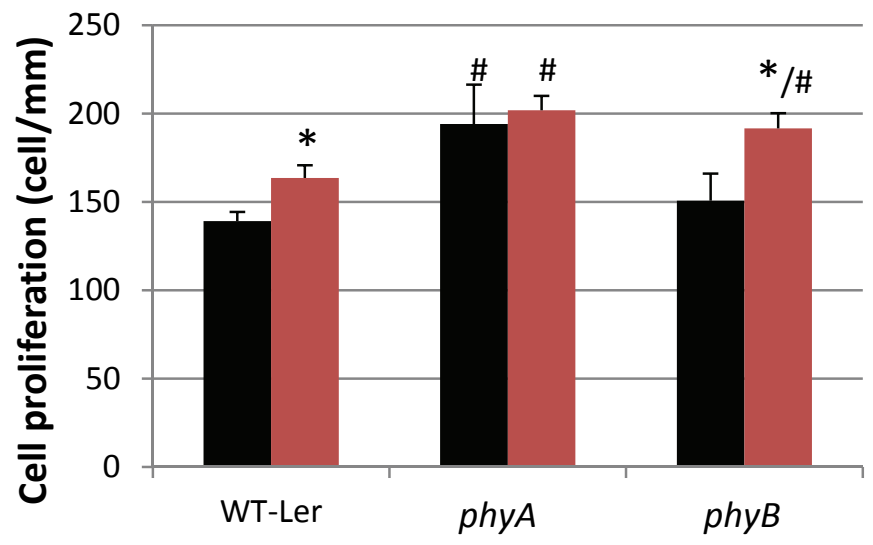

no photoactivation $\quad$ Red light photoactivation 


\section{$1 g$ RPM controls}

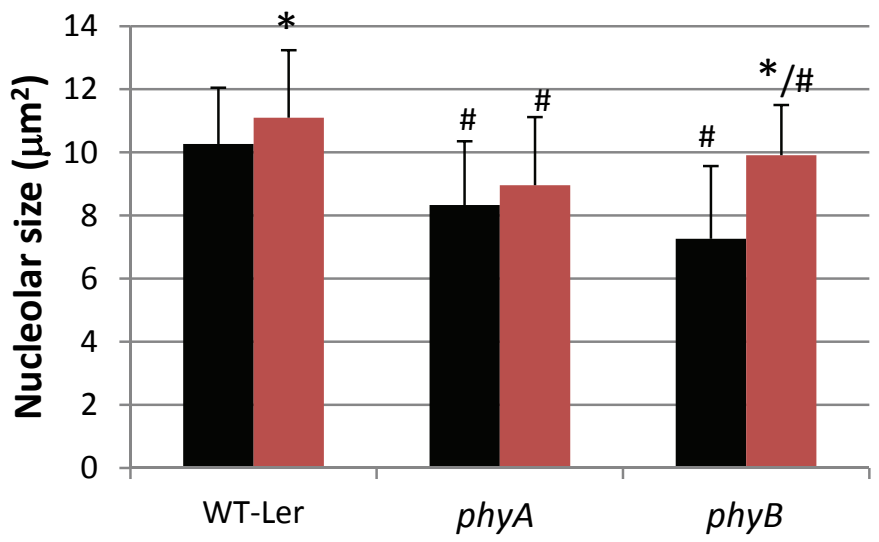

No photoacivation Red light photoactivation 
a) No photoactivation

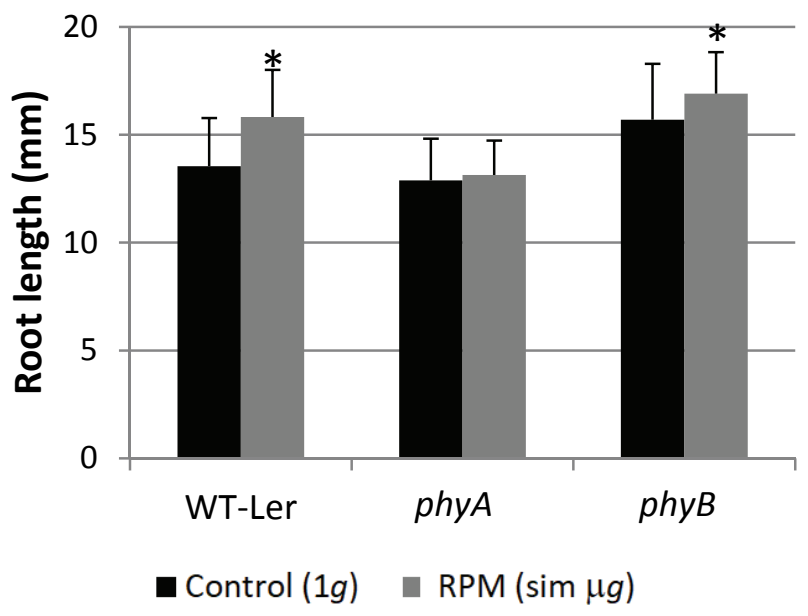

b) Red light photoactivation

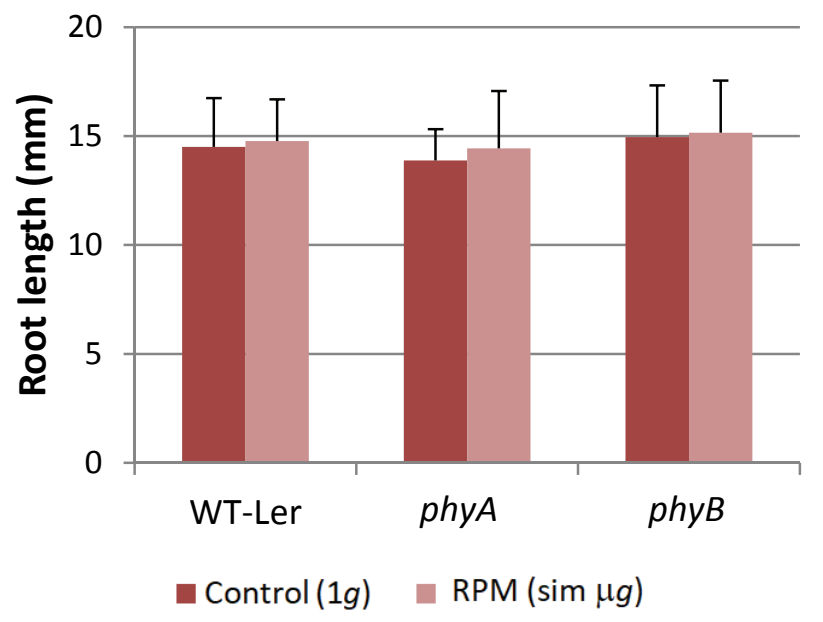




\section{a) No photoactivation}

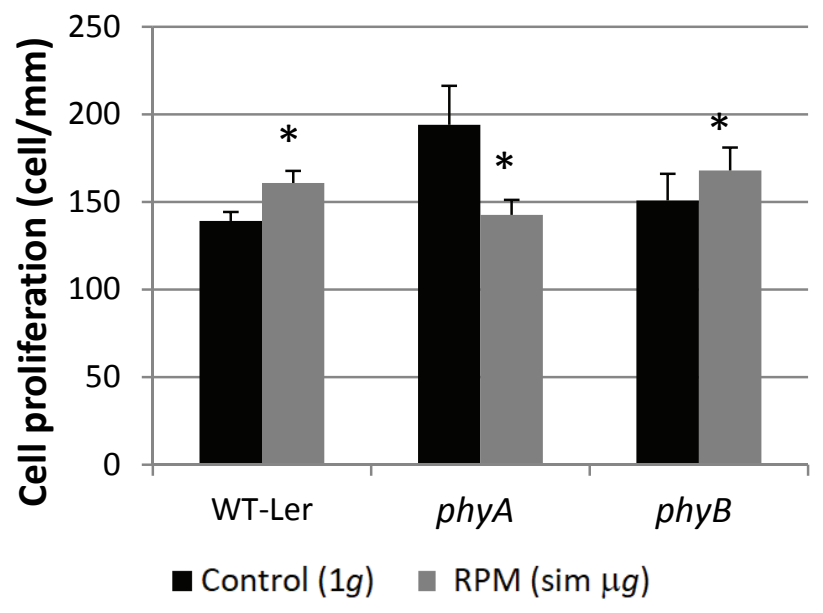

b) Red light photoactivation

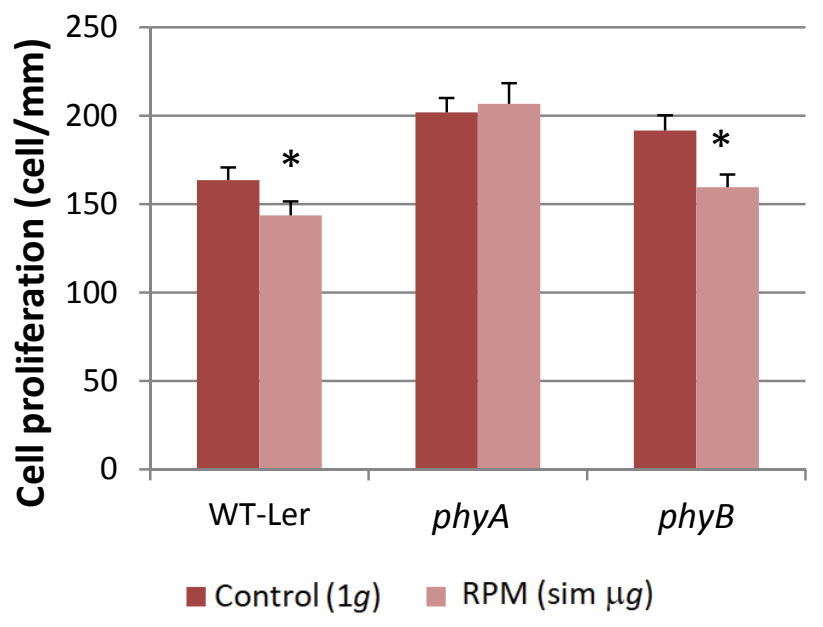




\section{RPM Experiment (Sim $\mu g)$}

a) No photoactivation b) Red light

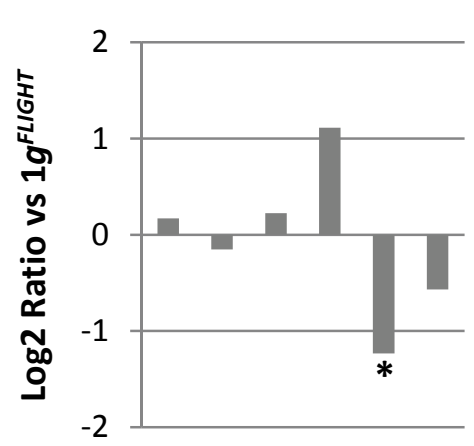

photoactivation
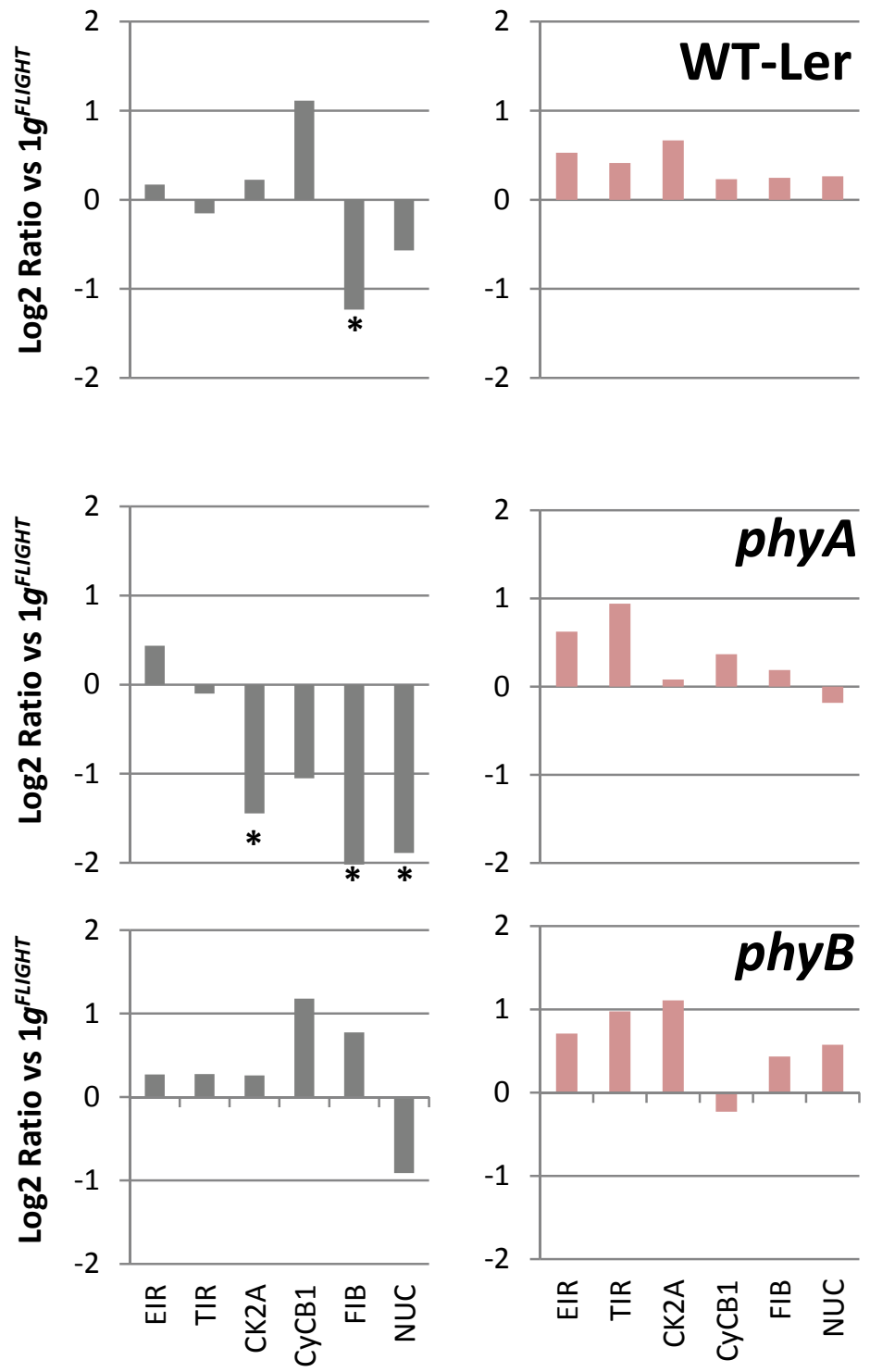
a) No photoactivation

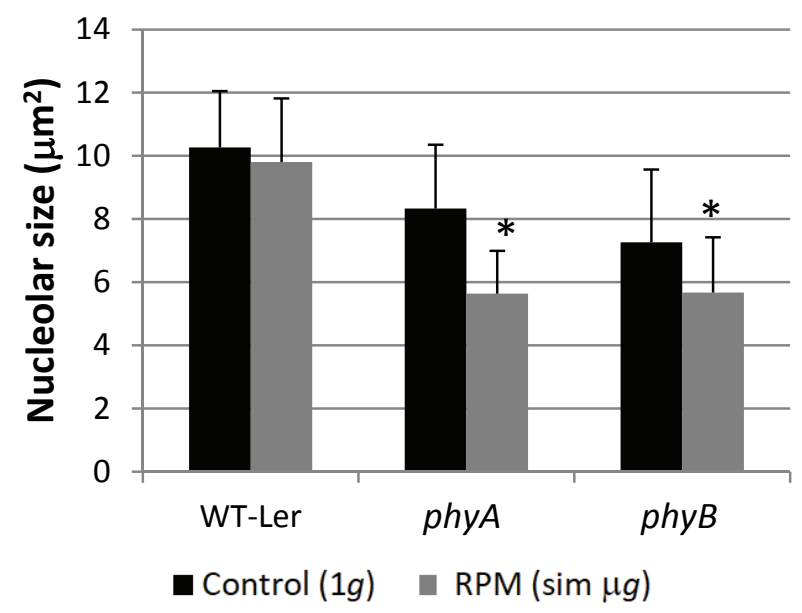

\section{b) Red light photoactivation}

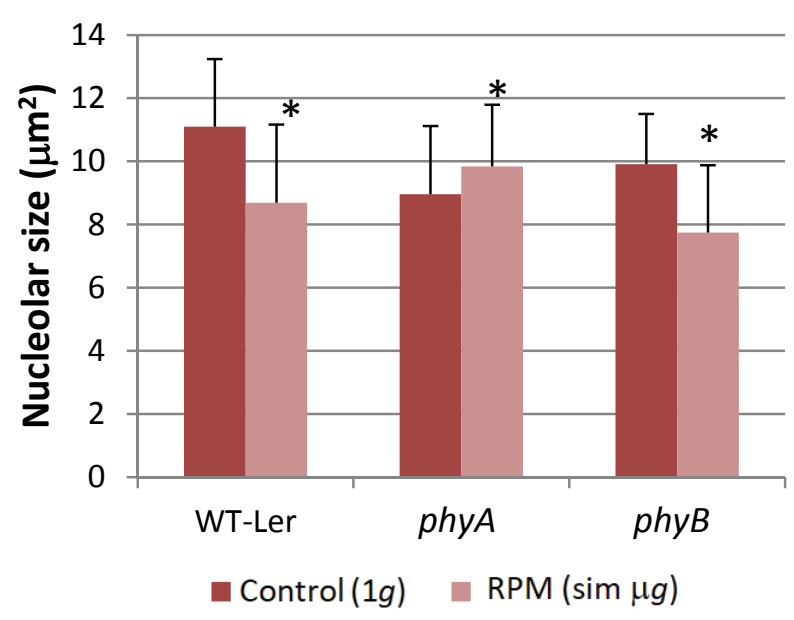


Table 1 Summary of the combined effects of microgravity (real - ISS or simulated - RPM) or partial gravity $(0.1 g, 0.3 g, 0.5 g$ on ISS) and red light photoactivation on functional parameters of root meristematic cells. Note the similar strategy in WT-Ler and phyB to deal with microgravity environments while $p h y A / p h y B$ mutants share a similar fractional gravity response.

\begin{tabular}{|c|c|c|c|c|c|c|c|}
\hline \multirow[t]{2}{*}{ WT-Ler } & \multicolumn{2}{|c|}{$\begin{array}{l}\text { Cell } \\
\text { Proliferation }\end{array}$} & \multicolumn{2}{|c|}{ Cell Growth } & \multirow{2}{*}{$\begin{array}{l}\text { Meristematic } \\
\text { Competence }\end{array}$} & \multirow[t]{2}{*}{ Auxin } & \multirow{2}{*}{$\begin{array}{l}\text { Root } \\
\text { length }\end{array}$} \\
\hline & Rate & Genes & Nucleolus & Genes & & & \\
\hline $\begin{array}{l}\text { ISS: Microgravity } \\
\text { +Red Light (1) }\end{array}$ & & 4 & & 4 & YES, fast & 4 & 4 \\
\hline $\begin{array}{l}\text { RPM: 1g control } \\
+ \text { Red Light (2) }\end{array}$ & 4 & & 4 & & YES, fast & & $\uparrow$ \\
\hline $\begin{array}{l}\text { RPM: Sim } \boldsymbol{\mu g} \\
\text { No Red Light (2) }\end{array}$ & 4 & $\uparrow$ & $\downarrow$ & $\downarrow$ & NO & $=$ & 4 \\
\hline $\begin{array}{l}\text { RPM: Sim } \boldsymbol{\mu g} \\
\text { + Red Light (1) }\end{array}$ & $\downarrow$ & $\uparrow$ & $\downarrow$ & $=$ & YES & $\uparrow$ & $=$ \\
\hline $\begin{array}{l}\text { ISS:Partial 0.1-0.5g } \\
\text { + Red Light (1) }\end{array}$ & & $=$ & & $=$ & YES & $\downarrow$ & $\uparrow$ \\
\hline
\end{tabular}

\begin{tabular}{|c|c|c|c|c|c|c|c|}
\hline \multirow[t]{2}{*}{ phyB mutant } & \multicolumn{2}{|c|}{$\begin{array}{l}\text { Cell } \\
\text { Proliferation }\end{array}$} & \multicolumn{2}{|c|}{ Cell Growth } & \multirow{2}{*}{$\begin{array}{l}\text { Meristematic } \\
\text { Competence }\end{array}$} & \multirow[t]{2}{*}{ Auxin } & \multirow{2}{*}{$\begin{array}{l}\text { Root } \\
\text { length }\end{array}$} \\
\hline & Rate & Genes & Nucleolus & Genes & & & \\
\hline $\begin{array}{l}\text { ISS: Microgravity } \\
\text { +Red Light (1) }\end{array}$ & & 4 & & 4 & YES, fast & 4 & 4 \\
\hline $\begin{array}{l}\text { RPM: 1g control } \\
\text { +Red Light }(2)\end{array}$ & 4 & & 4 & & YES, fast & & $\uparrow$ \\
\hline $\begin{array}{l}\text { RPM: Sim } \boldsymbol{\mu g} \\
\text { No Red Light (2) }\end{array}$ & 4 & $\uparrow$ & $\downarrow$ & $\downarrow$ & $\mathrm{NO}$ & $=$ & 4 \\
\hline $\begin{array}{l}\text { RPM: Sim } \boldsymbol{\mu g} \\
\text { + Red Light (1) }\end{array}$ & $\downarrow$ & $=$ & $\downarrow$ & $=$ & YES & $\uparrow$ & $=$ \\
\hline $\begin{array}{l}\text { ISS:Partial 0.1-0.5g } \\
\text { + Red Light (1) }\end{array}$ & & $\downarrow$ & & $\downarrow$ & YES, slow & $\downarrow$ & $\uparrow$ \\
\hline
\end{tabular}

\begin{tabular}{|c|c|c|c|c|c|c|c|}
\hline \multirow{3}{*}{ phyA mutant } & \multirow{2}{*}{\multicolumn{2}{|c|}{$\begin{array}{l}\text { Cell } \\
\text { Proliferation }\end{array}$}} & \multirow{2}{*}{\multicolumn{2}{|c|}{ Cell Growth }} & \multirow{3}{*}{$\begin{array}{l}\text { Meristematic } \\
\text { Competence }\end{array}$} & \multirow{3}{*}{ Auxin } & \multirow{3}{*}{$\begin{array}{l}\text { Root } \\
\text { length }\end{array}$} \\
\hline & & & & & & & \\
\hline & Rate & Genes & Nucleolus & Genes & & & \\
\hline $\begin{array}{l}\text { ISS: Microgravity } \\
\text { +Red Light (1) }\end{array}$ & & $=$ & & $\downarrow$ & YES & $=$ & 4 \\
\hline $\begin{array}{l}\text { RPM: 1g control } \\
\text { +Red Light (2) }\end{array}$ & $\uparrow$ & & $\uparrow$ & & YES, fast & & 4 \\
\hline $\begin{array}{l}\text { RPM: Sim } \boldsymbol{\mu g} \\
\text { No Red Light (2) }\end{array}$ & $\downarrow$ & $\downarrow$ & $\downarrow$ & $\downarrow$ & YES, slow & $=$ & $=$ \\
\hline $\begin{array}{l}\text { RPM: Sim } \boldsymbol{\mu g} \\
\text { + Red Light (1) }\end{array}$ & $\uparrow$ & $=$ & $\uparrow$ & $=$ & YES & $\uparrow$ & $=$ \\
\hline $\begin{array}{l}\text { ISS:Partial 0.1-0.5 } \\
+ \text { Red Light (1) }\end{array}$ & & $\downarrow$ & & $\downarrow$ & YES, slow & $\downarrow$ & $\uparrow$ \\
\hline
\end{tabular}

(1) Compared to $1 g$ control (red light). (2) Compared to $1 g$ - no red light. =: No clear variations.

4: Significant increase $(P<0,05) ; \uparrow$ : Low-significant increase $(P>0,05, \log 2$ Ratio $>.5)$;

$\downarrow$ : Significant decrease $(P<0,05) ; \downarrow$ : Low-significant decrease $(P>0,05$, Log2Ratio $<-.5)$ 


\section{Click here to access/download Supplementary Material \\ 2018_03 Paper_SG12_PhyAB Supplementary Material.pdf}




\section{Click here to access/download Supplementary Material 2018_03 Paper_SG12_PhyAB_FINAL- review6_TrackChanges.docx}

